\title{
DER FILM IM GEOGRAPHIEUNTERRICHT
}

\section{Georg Pool}

Der Unterrichtsfilm ist heute kein Unbekannter mehr. Seit seiner Einführung als Hilfsmittel des modernen Unterrichtes sind mehr als 30 Jahre vergangen. Am 8. Oktober 1922 hatte Prof. Dr. E. Rüst, Zürich, an der Tagung des Vereins Schweizerischer Gymnasiallehrer seine Leitsätze über den Unterrichtsfilm vorgetragen und diese wurden allgemein gutgeheißen. Dennoch ist bis heute die Diskussion über den Wert des Unterrichtsfilmes nicht abgeschlossen. Es sind weniger die grundsätzlichen als die Probleme methodisch-didaktischer Art, wie Fragen der Filmgestaltung für die verschiedenen Schulfächer, die immer wieder neu aufgeworfen werden.

Mit der äußerst raschen Entwicklung der Unterrichtsfilmproduktion, besonders im Ausland, gingen auch die Ansichten immer mehr auseinander, so daß es wünschbar erscheint, an dieser Stelle vom besonderen Standpunkt des GeographieUnterrichtes alle Fragen einer erneuten Prüfung zu unterziehen. Dabei wollen wir uns bewußt bleiben, da $\beta$ eine Einigung wohl nur über die wesentlichsten Punkte möglich ist, da bei der Beurteilung eines bestimmten Beispieles sich die persönlichen Meinungen nie auf einen Nenner bringen lassen werden. Das dürfte aber nur von Vorteil sein, da wir sonst rettungslos einem unerwünschten Schematismus verfallen.

Die von Rüst aufgestellten Forderungen gelten heute noch. Es sind die folgenden:

1. Der Film zeigt Vorgänge, bei denen die Be-zuegung wesentlich ist. Die besondere Eigenart des Filmes ist, daß er durch die rasche Folge der Einzelbilder die Bewegung zeigt, die nicht nur mechanische Bewvegung sein muß, sondern auch in der Spannung, im Rhythmus der Bildfolge gelegen sein kann. Die besondere psychophysiologische Situation, in die der Zuschauer durch das Filmgeschehen versetzt wird, kann hier in positivem Sinne ausgenützt werden. Ein Beispiel dafür sei der "Paricutin-Film" (Z 301, Ba 205, Be 255, SG 159).

2. Der Unterrichtsfilm gehört wie das Lichtbild in den Unterricht. Dieser Leitsatz erscheint selbstverständlich, und doch muß er immer wieder erneut betont werden. Wie es niemandem einfallen dürfte, Lichtbilder zu einer andern Zeit als in der Unterrichtsstunde, zu welcher sie gehören, zu zeigen, so darf auch der Unterrichtsfilm nicht zu einer anderen Zeit als in der Unterrichtsstunde vorgeführt werden, wenn er dem Schüler nicht zur Unterhaltung mit mehr oder weniger Belehrung dienen soll.

3. Der Filminhalt ist der Schulstufe anzupassen. So wie das Lehrbuch den gleichen Unterrichtsgegenstand der Schulstufe angepaßt behandelt, so soll auch der Unterrichtsfilm in seinem Inhalt und in der Wiedergabe angepaßt sein. Es ist völlig sinnlos, einer Klasse der Unterstufe z. B. den Film "Zyklonenbildung auf der Wetterkarte" (Z 381) zu zeigen, da die Schüler das Geschehen in der Abstraktion des Trickfilmes nicht erfassen können. Umgekehrt wird ein Schüler der Oberstufe für einen Film wie "Die Schleuse" (Z 103, Ba 28) wohl nur ein Lächeln übrig haben, da er sich das Geschehen auch ohne Film sehr gut vorstellen kann.

4. Der Unterrichtsfilm ist nur dort einzusetzen, wo er den ïbrigen Unterrichtshilfsmitteln überlegen ist. Es gibt wohl kein anderes Fachgebiet, wie gerade die Geographie, wo dieser Leitsatz besonderes Gewicht erhält. Es wird Aufgabe der nachfolgenden Ausführungen sein, namentlich diese Frage zu erörtern.

Zunächst seien nun die Möglichkeiten des Filmes aufgezeigt und der Versuch einer Klassifikation der in Frage kommenden Filmtypen unternommen; denn es gibt nicht den Unterrichtsfilm für Geographie.

1. Die technischen Möglichkeiten: Der Film kann, wie kein anderes Anschauungshilfsmittel zwei sonst unveränderliche Erscheinungsgruppen: Räumliche und zeitliche, beliebig ändern. Nicht nur kann die Aufnahmekamera jeden beliebigen Standort einnehmen, sie kann auch mittels des Teleobjektives und der Lupe den Raum in allen seinen Dimensionen erschließen. Mittels der Zeitraffung oder der Zeitlupe kann der Faktor Zeit verkleinert oder vergrößert werden, so daß sich Vorgänge der Beobachtung erschließen, die sonst nicht veranschaulicht werden können.

Im Trickflm (Zeichentrick, Modelltrick) ist eine weitere Möglichkeit geboten, die mit Naturaufnahmen kombiniert, wesentlich zum Verständnis beitragen kann. 
Wo die Farbe (Farbenfilm) ein wesentliches Element des Bildes ist, kann auch sie zur Darstellung gelangen. Leider muß aber heute noch einschränkend gesagt werden, daß die Farbentreue in den Kopien nicht immer restlos befriedigt. Auch die Preisfrage soll nicht verschwiegen werden. In den meisten Fällen sind es aber die Formelemente, denen die Hauptbedeutung zukommt, so da $\beta$ meistens mit dem Schwarz-weiß-Film auszukommen ist. Es ist indes zu hoffen, daß in absehbarer Zeit auch die Frage des Farbenfilmes eine allgemein befriedigende Lösung finden wird.

Der Ton kann, soweit er im geographischen Unterrichtsfilm eine Rolle spielt, mittels verschiedener Verfahren mit dem Geschehen synchron wiedergegeben werden. Es gibt aber nur wenige geographische Sujets, wo der Ton von Bedeutung sein dürfte. Somit genügt in der Regel für den geographischen Unterrichtsfilm der S'tummfilm vollauf. Auf den Tonfilm als kommentierter Film wird an anderer Stelle einzutreten sein.

2. Möglichkeiten der Filmgestaltung. Da der Unterrichtsfilm möglichst objektiv sein muß, ist er seinem Wesen nach ein Dokumentarfilm. Und doch unterscheidet er sich von diesem, indem die Wiedergabe des Geschehens je nach den Bedürfnissen des Unterrichtes durch die Anwendung besonderer technischer Möglichkeiten modifiziert wird. Es können räumliche und zeitliche Zusammenhänge neu konstruiert oder gelöst werden, die der absoluten Wirklichkeit nicht entsprechen. Es wird damit aber keine Fälschung der Wirklichkeit begangen, sondern lediglich die Anschaulichkeit oder das bessere Verständnis angestrebt.

Je nach dem Inhalt des Unterrichtsfilmes werden verschiedene Typen der Filmgestaltung und damit des Filmschaffens möglich, angefangen bei Kurzfilmen über eine bestimmte Erscheinung bis zu Übersichten über weiteste Räume durch das Aneinanderhängen mehr oder weniger zusammenhängender Szenen in sogenannten Übersichtsfilmen. Während die erstgenannten Kurzfilme in bezug auf den Inhalt kaum einer nennenswerten Kritik unterliegen werden, wird die zweite Art, die Übersichtsfilme, wohl nie allgemein befriedigende Resultate ergeben. Bei der Beurteilung solcher Filme spielt das individuelle Empfinden eine ausschlaggebende Rolle.

$\mathrm{Da} \beta$ alle Filme wahr sein müssen, also nicht durch «Montage 》 Fälschungen des Sachverhaltes vermittelt werden dürfen, braucht wohl nicht betont zu werden. In diesem Sinne muß auch das menschliche Handeln im Film《wahr» sein, d. h. darf nicht gestellt oder einstudiert wirken.

Endlich muß der Unterrichtsfilm auch in dem Sinne nicht die Vollständigkeit eines Dokumentarfilmes aufweisen, da $\beta$ er sich nur auf das Wesentliche beschränkt, nämlich nur auf das, was einzig mittels des Filmes gezeigt werden kann. Damit unterscheidet sich der Unterrichtsfilm aber auch vom Lehrfilm, der den Lehrer ersetzen soll.

Nachdem die Möglichkeiten summarisch aufgezeigt worden sind, seien nun die besondern Forderungen des Faches Geographie genannt. Wie schon angedeutet, kann es sich dabei nur um die eingangs zitierten allgemeinen Forderungen handeln, unter besonderer Berücksichtigung des Faches.

Vorerst seien die Forderungen genannt, die an die Kurzfilme gestellt werden müssen. In einem solchen Streifen soll der darzustellende Vorgang mit den besten technischen Hilfsmitteln dargestellt werden, wobei eine Kombination von Naturund Trickaufnahmen in den meisten Fällen den Wert des Filmes wesentlich erhöht.

Der Kurzfilm soll sich aber nur auf einen Vorgang beschränken und nicht eine - lehrbuchartige Abhandlung aller Aspekte des gezeigten Vorganges bieten. In dieser bewußten Vereinfachung liegt auch die Problematik des Kurzfilmes. Es werden niemals alle am Geschehen beteiligten Faktoren dargestellt werden können. Wieweit indes dieser Mangel in Kauf genommen werden kann, hängt von der Bereitschaft und den Möglichkeiten ab, die dem Lehrer zur Verfügung stehen, um ihn, sei es 
durch andere Anschauungsmittel, sei es durch seinen Kommentar auszugleichen. Die Diskussion gerade solcher Mängel kann aber die Auswertung eines Kurzfilmes wesentlich befruchten und damit zur Vertiefung des Geschauten beitragen. Da auch der Unterricht selbst nicht alle Aspekte gleichzeitig behandelt, so wird eventuell die einem Kurzfilm vorgeworfene Unvollständigkeit geradezu sein Vorteil.

Die überwiegende Mehrzahl geographischer Filme wird wohl zu den sogenannten Übersichtsfilmen zu zählen sein. Filme für den länderkundlichen Unterricht, Filme, die kulturgeographische oder wirtschaftsgeographische Einheiten zur Anschauung bringen, sind am meisten verlangt. Welche Forderungen sind an einen solchen Film zu stellen?

Diese Frage ist am schwersten zu beantworten. Selbst das Ausland hat sie nicht einheitlich beantwortet. Daher sei versucht, die bisher im Ausland gegebenen Antworten zu diskutieren.

Nennen wir zunächst das negative Beispiel. In den entsprechenden Filmen wird mehr oder weniger dem Gange eines Lehrbuches folgend gleichsam Kapitel um Kapitel ein Land dargestellt. Beginnend mit der Lage, wird anschließend die Naturlandschaft, dann die Kulturlandschaft vorgeführt. Durch Trickteile werden anders nicht darstellbare Zusammenhänge veranschaulicht, die statistischen Daten oft durch «lebende Statistik 》gezeigt. Eine andere Art sind zu einem Film vereinigte «Lichtbilder », ohne organischen Zusammenhang. Daß diese Art 《geographische Filme» keine Unterrichtsfilme sind, ergibt sich aus der Tatsache, daß sie entweder eine solche Fülle von Stoff vermitteln, daß der Zuschauer am Schluß meist nicht mehr weiß, was er am Anfang gesehen hat, oder aber daß sie wegen der Sprunghaftigkeit eher verwirren als belehren.

Einen anderen Typus stellen die «Reisefilme»dar. Hier wird im Film einc Reise durch ein Land gezeigt, wobei die Reiseroute als «roter Faden» dient. Bei methodisch gutem Aufbau können solche Filme bereits als wertvoll bezeichnet werden.

Wieder andere Produzenten stellen den Menschen in den Mittelpunkt ihres Filmes und zeigen am Leben eines Einzelnen oder seiner Familie den Lebensraum und «the way of life » im betreffenden Lande. Damit wird der Versuch unternommen, der modernen Forderung nach «Human Geography » Genüge zu tun.

In Deutschland wird die Auffassung vertreten, daß im Erlebnisflm der hauptsächliche schulische Wert liegt.

HornberGer ${ }^{1}$ definiert den Erlebnisfilm wie folgt: „Es handelt sich dabei um einen Film, der ein Geschehen in der Landschaft aus sich heraus und als Ganzes gestaltet. Dazu müssen kausale Verknüpfungen und funktionale Wechselbeziehungen der natürräumlichen und kulturräumlichen Kräfte zu einem Geflecht verwoben sein, das alle Spannungsmomente enthält und in unmittelbarer lebendiger Anschauung den Betrachter das Bild und die Vorgänge in der Landschaft nacherleben läßt. Möglichst wenig lehrhaftes und möglichst kein Trick soll dieses Erlebnis stören. Es wird dabei nicht auf eine systematische länderkundliche Erfassung eines Raumes Wert gelegt, sondern versucht, an einem Ausschnitt die landschaftlichen Zusammenhänge erlebnishaft zu gestalten."

Es darf nicht verschwiegen werden, daß die Schaffung gerade solcher Erlebnisfilme an den Gestalter des Filmes die größten Anforderungen stellt und daß bisher die Forderung nur ungenügend erfüllt wurde.

Damit sind die wichtigsten Formen länderkundlicher Filme gekennzeichnet. Überprüfen wir diese Filme nach den eingangs genannten Leitsätzen, so ist nicht schwer zu erkennen, daß wohl in der Richtung der deutschen Auffassung der richtige Weg zu suchen ist.

Hier ist aber auch noch ein weiterer Faktor zu nennen, der von größter Bedeutung ist: der Kommentar des Lehrers. Auch der beste Erlebnisfilm wird auf die Schüler ohne einen entsprechenden Kommentar des Lehrers nicht die Wirkung

1 Hornberger, T.: Lichtbild und Film im Unterricht der Geographie, Geographische Rundschau, 4, 1952, Nr. 12. 
ausüben wie mit einem Kommentar. Die logische Folge wäre nun, daß der Unterrichtsfilm einen wohldurchdachten und ausgefeilten Kommentar enthalten sollte, damit jene maximale Wirkung erzielt werden kann. Dieser Schluß erweist sich aber als Trugschluß wie Versuche in verschiedenen Ländern eindeutig gezeigt haben 2.

Es erübrigt sich daher, ausführlicher auf dieses Problem einzutreten. Nur einige wenige Tatsachen seien genannt, die in diesem Zusammenhange in Erwägung zu ziehen sind. Der beste Kommentar wird der der Schulstufe und dem Schultypus angepaßte Kommentar sein. Dies bedeutet aber, daß zu jedem Film mehrere Kommentare nötig wären. Angesichts des Umstandes, daß der Unterrichtsfilm an s:ch schon ein relativ teures Unterrichtshilfsmittel ist, würde durch dịe Herstellung verschiedener Tonfassungen der Film nur weiter verteuert. Es besteht allerdings im Magnetton-Verfahren eine billige Möglichkeit, den Kommentar eines Filmes von Fall zu Fall neu zu sprechen. Beim Magnetton-Verfahren wird wohl meist der Klassenlehrer den Film «besprechen » müssen. Damit bietet es doch keinen Vorteil, denn die mechanische Wiedergabe des vom Lehrer gesprochenen Textes nimmt diesem viel von seiner Unmittelbarkeit. Der Stummfilm aber kann in der gleichen Fassung meist auf verschiedenen Stufen gezeigt und jeweils entsprechend kommentiert werden.

Es wird mit der Vorführung des Stummfilmes der direkte Sprechkontakt zwischen Lehrer und Schüler nicht unterbrochen, weshalb sogar angelsächsische Unterrichtsfilmstellen zu nachfolgendem Schlusse gelangt sind: "As a result of the investigations the Committee concluded that within the limits of this experiment it would appear that the silent film with teachers commentary is a more efticative aid than either the sound or the silent version of the same Film !" 3

Anders verhält es sich beim Lehrfilm; doch wurde bereits darauf hingewiesen, daß ein Unterrichtsfilm kein Lehrfilm sei.

$\mathrm{Zu}$ den «geographischen Filmen» (im weitern Sinne) werden auch Filme gezählt, die monographisch die Gewinnung oder Herstellung einer Ware, eines Rohstoffes zeigen. Gerne werden solche Filme als «wirtschaftsgeographische Filme 》 bezeichnet. Sicher werden auch diese da und dort Anwendung finden können, doch eignen sie sich wohl in erster Linie für den Warenkunde-Unterricht an entsprechenden Schulen. Bei den eigentlichen wirtschaftsgeographischen Filmen werden die gleichen Forderungen zu stellen sein wie bei den Übersichtsfilmen, d. h. auch sie werden die Beziehungen zwischen Natur und Mensch in den Vordergrund stellen müssen, nicht die Ware.

Zusammenfassend halten wir fest, daß der geographische Unterrichtsfilm grundsätzlich den gleichen Forderungen entsprechen muß wie jeder andere, wobei die in der Wechselwirkung Natur - Mensch beruhende Spannung wohl am besten durch Erlebnisfilme dargestellt werden kann. Analog sollen Landschaftsfilme die besondere Dynamik, den Rhytmus der Landschaft zeigen, wobei aber der Film immer nur dort eingesetzt werden darf, wo er dem Lichtbild überlegen ist.

Die Verwendung des Filmes im Unterricht erfolgt grundsätzlich nach den gleichen methodischen Gesichtspunkten, wie die Verwendung des Lichtbildes. Dazu kommt aber, daß beim Film der Lehrer sich für den Kommentar eingehender vor.bereiten muß als beim Lichtbild. Besondere Aufmerksamkeit ist dabei der Szenenfolge zu schenken, da dem Lehrer im Unterschied zum Lichtbild nicht unbeschränkte Zeit für den Kommentar zur Verfügung steht. Denn es muß in der kurzen Zeit des Szenenablaufes alles gesagt werden, was wichtig ist. Eine ausführlichere Auswertung des Gesehenen erfolgt meist nach der Vorführung des Filmes. Die Schüler sind also auf die wichtigen Punkte am jeweils richtigen Ort hinzuweisen.

2 Pool, G.: Tonfilm - Stummfilm, Beitrag zur Frage ihres Wertes als Unterrichtshilfsmittel. Der Unterrichtsfilm, 3, $1952, \mathrm{Nr} .4$.

3 Mcintosi, D. M.: A Comparison of Efficasy of Sound and Silent Films as Teaching Aids, Research Publication No. 3 der Scottish Educational Film Association, 1947. 
Bei Kurzfilmen kann durch wiederholtes Vorführen des Streifens das Gesehenc vertieft werden, oder es kann der Film auch zur Zusammenfassung verwendet werden. Anzustreben ist, daß die Schüler den Film nicht nur passiv «anschauen 》, sondern aktiv bei der Auswertung der Szenen mitmachen.

Völlig falsch wäre es, einen Film ohne jeden Kommentar zu zeigen, wie es auch abwegig ist, wenn die Vorführung besonders der Übersichtsfilme nicht im Unterricht entsprechend vorbereitet wird. Der Schüler soll mit den durch den Film zu veranschaulichenden Fragen vertraut sein, sie nicht erst während der Vorführung selbst suchen müssen. Dazu wird auch der Unterrichtsfilm nur in den seltensten Fällen genügend lange Szenen aufweisen, trotz der Tatsache, daß in den Unterrichtsfilmen die Szenen gegenüber «Kulturfilmen » länger sind. Der rasche Szenenwechsel bei «Kulturfilmen » ist cin.oft angewandtes Mittel, um filmische Spannung zu schaffen. Die größere Länge der Szenen hat dem Unterrichtsfilm das Prädikat « langweilig 》 eingetragen; diese Eigenschaft wird aber von keiner Klasse empfunden, die einen Unterrichtsfilm aktiv auswertet. Nur zu oft wird, besonders bei Kurzfilmen, der Fall eintreten, daß die Szenenlänge nicht ausreicht, um gegebenentalls mehrere Fragestellungen gleichzeitig beantworten zu können. Dann wird eine $z$ weite Vorführung unerläßlich sein.

$\mathrm{Da}$ ein Film auch zur Einführung in ein neues Arbeitsgebiet verwendet werden kann, sei nur der Vollständigkeit halber erwähnt. Dabei wird jeweils dem Schüler überlassen, «etwas» $z u$ schen. «Filmgewohnte 》Schüler werden dann auch wirklich allerlei herausfinden, oft mehr sehen, als der Lehrer selbst gesehen haben mag! Gegen solche «Überraschungen» gibt es nur ein Mittel: den Film selbst sehr gründlich anzusehen, bevor er der Klasse gezeigt wird.

Woher können Unterrichtsfilme bezogen werden? Auf diese Frage sei hier zuletzt kurz eingetreten. Ideal wäre es, wenn jede Schule über eine eigene Filmothek verfügen würde, wie dies in einigen Schulen bereits der Fall ist. Speziell für die Kurzfilme sollte dies der Fall sein. Die längeren «Übersichtsfilme » werden wegen ihres relativ hohen Anschaffungspreises wohl meist von den bestehenden Unterrichtsfilmstellen leihweise bezogen werden.

Für das Gebiet der Schweiz stehen zur Zeit mehrere Filmotheken zur Verfügung. Diese sind im Rahmen der Vereinigung Schweizerischer Unterichtsfilmstellen (VESU) untereinander verbunden und arbeiten nach den gleichen, hier wiedergegebenen Grundsätzen.

Um eine rasche und reibungslose Bedienung der Schulen sicherzustellen, wurde das Gebiet der Schweiz auf die einzelnen Unterrichtsfilmstellen verteilt. Die Kantonale Lehrfilmstelle Basel bedient die Kantone Basel-Stadt und Land, die Schulfilmzentrale Bern, Bern, die Kantone Bern, Solothurn, Uri, Schwyz, Unterwalden, Zug, Tessin und die welschen Kantone, die Schweizerische Arbeitsgemeinschaft für Unterrichtskinematographie (S.JFU), Zürich, die Kantone Zürich, Aargau, 'Thurgau, Schaffhausen, Appenzell, Glarus und Graubünden, während der Kanton St. Gallen eine eigene Kantonale Lehrfilmstelle besitzt.

Die Bezugsmöglichkeiten sind bei den Filmstellen in Bern und Zürich die gleichen, d. h. die Schulen bestellen bei der zuständigen Stelle die Filme rechtzeitig und erhalten diese gegen eine Leihyebühr, die je nachdem ob die Schule Mitglied ist oder nicht abgestuft ist. Die Kantonalen Lehrfilmstellen liefern an die ihnen zu. gewiesenen Schulen gratis. Ein Abonnementssystem ermöglicht allen Schulen, bei allen Unterrichtsfilmstellen $\mathrm{zu}$ den gleichen Bedingungen Filme zu beziehen.

Über die zur Zeit in den schweizerischen Unterrichtsfilmstellen verfügbaren Filme orientiert das beigelegte Filınverzeichnis.

LE FILM DANS L'ENSEIGNEMENT DE LA GÉOGRAPHIE

Il y a plus de 30 ans que le prof. Dr. E. Rứst de Zurich, a établi les principes de l'emploi du film dans l'enseignement; ces principes ont gardé leur valeur. L'un d'eux, qui n'admet l'emploi du 
film que lorsqu'il est supérieur aux autres moyens, est ici considéré au point de vue de la géographie. Après l'examen des possibilités techniques du film et de son établissement, vient la question des exigences posées par l'enseignement de la géographie. Le film documentaire peut, en "court métrage», traiter d'un fait particulier, ou en evue générale» montrer les relations entre faits et paysages géographiques. On étudie ensuite les conditions spéciales au sens des principes énoncés plus haut. Le problème est difficile à résoudre. L'Allemagne envisage des afilms vécus» qui montrent la vie dans un paysage considéré comme un tout. Malheureusement le nombre des films géographiques est encore très modeste. Pour finir on montre les possibilités de créer des films en Suisse.

\section{IL FILM NELLINSEGNAMENTO DELLA GEOGRAFIA}

Più di trennt'anni or sono il Prof. Dr. E. Rūst di Zurigo aveva formulato i criteri che avrebbero dovuto ispirare il film scolastico: essi hanno lo stesso valore ancora oggigiorno. Per principio si serebbe dovuto ricorrere al film soltanto in quei casi in cui esso si fosse dimostrato nettamente superiore agli altri mezzi didattici: è soprattuto questo punto di vista che vien discusso nel presente lavoro. Tenuto calcolo dei progressi tecnici conseguiti e le possibilità offerte dal film ci si domanda quasi siano $\mathrm{i}$ requisiti che si possono richiedere al film scolastico perchè diventi di ausilio per l'insegnamento della geografia. Esso può avere il carattere di un „documentario“, o la forma di un "cortometraggio “ trattante un determinato fenomeno o ancora la veste di un "film panoramico" che dà una visione su variazioni e relazioni di paesi e paesaggi, dove sempre esso deve rispettare $i$ requisiti enunciati nell'introduzione. La Germania per esempio cerca la soluzione in un tipo di film che rappresenta un avvenimento nel quadro naturale del suo paesaggio con cui è intimamente legato. Purtroppo il numero dei buoni film geografici è ancora esiguo. In conclusione si accenna alle possibilità di acquisto di film scolastici nella Svizzera.

\section{REGIONALBESCHREIBUNGEN DER SCHWEIZ ALS GRUNDLAGE GEOGRAPHISCHER LANDESAUFNAHME UND LANDESPLANUNG}

Einer Aufforderung der Delegiertenversammlung der VSGG folgend, eine Grundlage zur Diskussion der vom Unterzeichneten 1951 zur Schaffung empfohlenen geographischen Gebietsbeschreibungen (GB) der Schweiz vorzubereiten, sind im folgenden einigen Gedanken wiedergegeben, die einer allgemeinen Aussprache dienen mögen. ${ }^{1}$

Ausgangssituation. Immer wieder stößt man in Ämtern, bei Verbänden, Privaten, in Schulen und auch in der Forschung auf den Mangel an orts- und landeskundlicher Dokumentation, der nicht nur die Verwaltungs- und Erziehungsarbeit wie das private Schaffen (des Ingenieurs, Geschäftsmannes usw.) empfindlich erschwert, sondern nicht selten auch wirtschaftliche, politische, technische, bauliche u.a. Fehlentscheide bedingt und der daher dringend Abhilfe verlangt. Dieser Mangel hat schon früher zu Versuchen der Schaffung von GB als Grundlagen der Verivaltung, Schule, Wirtschaft und Politik geführt. Sie leben neuerdings gerade in Ländern wieder auf, die schwere Kriegsbelastungen tragen. In der Schweiz belegen mindestens seit Beginn des 19. Jahrhunderts Werke wie „Gemälde der Schweiz“ (Kantonsbeschreibungen), Bezirks-, Amts- und Ortskunden ein andauerndes Bedürfnis, das jedoch nie zureichend befriedigt wurde. Der Grund hiefür dürfte zweifellos darin zu suchen sein, daß die bisherigen Unternehmen zu wenig systematisch, umfassend und allgemein verwendbar organisiert und durchgeführt wurden und daher auch nicht die generelle Unterstützung seitens der Öffentlichkeit fanden.

Der unbestreitbare Tatbestand der Wünschbarkeit einerseits und das Fehlen wirklich allseits befriedigender orts- und landeskundlicher Orientierungsmittel bezw. $\mathrm{GB}$ andrerseits gibt nun aber gerade der Geographie eine Chance: sich durch die Anregung, Planung und Schaffung solcher Beschreibungen in die Dokumentation einzuschalten. Sie vermöchte damit nicht nur der Öffentlichkeit einen unschätzbaren Dienst zu leisten, sondern sich selbst eine verbesserte Stellung - und ihren Vertretern gegebenenfalls vermehrte praktische Tätigkeit - zu gewinnen. Die Geographie besitzt

1 E. Dal V.esco ed E. WinkLer: La Geografia, Disciplina di pubblica utilità. Geographica Helvetica VI, 1951, 47-48. - F. JÆGGER: Projekt zur Erforschung aller Gemeinden der Schweiz. (Manuskript) Basel 1944. - H. ONDE: La géographie dans le cadre de la commune. Geographica Helvetica IV, 1949, 111-114. - E. W INKLER: Die Geographie in der schweizerischen Landesplanung. Schweiz. Geograph 21, 1942/43. Ders.: Zur Frage einer kulturgeographischen Landesaufnahme der Schweiz. (Manuskript eines Vortrags, gehalten am 9. Juni 1949 an der Universität Bern). Diese wenigen Hinweise seien angebracht, um daran zu erinnern, daß das im Titel genannte Thema in der jüngsten Zeit von verschiedenen Autoren auch in der Schweiz ventiliert worden ist, abgesehen davon, $\mathrm{da} B$ es in Nachbarländern, z. B. in Deutschland, bereits teilweise Realisierung fand. $\mathrm{Da} B$ übrigens in der Schweiz in dieser Beziehung ein reiches Material namentlich an geographischen Hochschulinstituten der Erschließung auch für die Öffentlichkeit harrt, sei in diesem Zusammenhang nur angedeutet. 